\title{
Open Journal of Mathematical
} Optimization

Zhou Wei, Michel Théra \& Jen-Chih Yao

Characterizations of Stability of Error Bounds for Convex Inequality Constraint Systems

Volume 3 (2022), article no. 2 (17 pages)

https://doi.org/10.5802/ojmo.13

Article submitted on July 2, 2021, revised on January 21, 2022,

accepted on February 22, 2022.

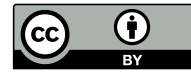

This article is licensed under the

Creative Commons AtTribution 4.0 International LiCEnse.

http://creativecommons.org/licenses/by/4.0/ 


\title{
Characterizations of Stability of Error Bounds for Convex Inequality Constraint Systems
}

\author{
Zhou Wei \\ College of Mathematics and Information Science, Hebei University, Baoding 071002, China \\ weizhou@hbu.edu.cn \\ Department of Mathematics, Yunnan University, Kunming 650091, China \\ wzhou@ynu.edu.cn \\ Michel Théra \\ XLIM UMR-CNRS 7252, Université de Limoges, Limoges, France \\ Federation University Australia, Ballarat \\ michel.thera@unilim.fr \\ Jen-Chih Yao \\ Research Center for Interneural Computing, China Medical University Hospital \\ China Medical University, Taichung 40402, Taiwan \\ yaojc@mail.cmu.edu.tw
}

\begin{abstract}
In this paper, we mainly study error bounds for a single convex inequality and semi-infinite convex constraint systems, and give characterizations of stability of error bounds via directional derivatives. For a single convex inequality, it is proved that the stability of local error bounds under small perturbations is essentially equivalent to the non-zero minimum of the directional derivative at a reference point over the unit sphere, and the stability of global error bounds is proved to be equivalent to the strictly positive infimum of the directional derivatives, at all points in the boundary of the solution set, over the unit sphere as well as some mild constraint qualification. When these results are applied to semi-infinite convex constraint systems, characterizations of stability of local and global error bounds under small perturbations are also provided. In particular such stability of error bounds is proved to only require that all component functions in semi-infinite convex constraint systems have the same linear perturbation. Our work demonstrates that verifying the stability of error bounds for convex inequality constraint systems is, to some degree, equivalent to solving convex minimization problems (defined by directional derivatives) over the unit sphere.
\end{abstract}

Digital Object Identifier 10.5802/ojmo.13

Keywords Local and global error bounds, Stability, Convex inequality, Semi-infinite convex constraint systems, Directional derivative.

Acknowledgments The first author was supported by the National Natural Science Foundation of China (grant 11971422) and CAS "Light of West China" Program, and by Jointed Key Project of Yunnan Provincial Science and Technology Department and Yunnan University [No. 2018FY001014] and Program for Innovative Research Team (in Science and Technology) in Universities of Yunnan Province [No. C176240111009]. Research of the second author benefited from the support of the FMJH Program PGMO and from the support of EDF.

\section{Introduction}

Our main goal in this paper is to study error bounds of a single convex inequality and semi-infinite convex constraint systems and to provide characterizations of stability of local and global error bounds under perturbations. Theory of error bounds can be traced back to the pioneering work by Hoffman [22] for systems of affine functions in which it has been proved that for a given matrix $A$ and a vector $b$, the distance from $x$ to the polyhedral set $\{u: A u \leq b\}$ is bounded above by some scalar constant (depending on $A$ only) times the norm of the residual error $\left\|(A x-b)_{+}\right\|$, where for any vector $z,(z)_{+}$denotes the positive part of $z$.

Hoffman's result was extensively and intensively studied by Robinson [48], Mangasarian [40], Auslender and Crouzeix [2], Pang [44], Lewis and Pang [36], Klatte and Li [30], Jourani [29], and there have been important developments of various aspects of error bounds for convex and nonconvex functions in recent years. We refer

() Zhou Wei \& Michel Théra \& Jen-Chih Yao;

licensed under Creative Commons License Attribution 4.0 International

(JMO Volume 3 (2022), article no. 2 
the readers to bibliographies $[3,4,6,9,14,15,17,18,19,23,27,34,37,41,43,45,52]$ and references therein for the summary of the theory of error bounds and their various applications for more details.

Error bounds have been applied to the sensitivity analysis of linear programs (cf. [47, 49]) and to the convergence analysis of descent methods for linearly constrained minimization (cf. $[20,21,28,38,51])$. In addition, it is proved that error bounds play an important role in the feasibility problem of finding a point in the intersection of a finite collection of closed convex sets (cf. $[7,8,9])$ and have an application in the domain of image reconstruction (cf. [13]). Also, error bounds are extensively discussed in connection with weak sharp minima of functions and metric regularity/subregularity as well as Aubin property/calmness of set-valued mappings (cf. $[1,6,10,11,12,19,25,26,31,32,48,54,55]$ and references therein).

Since real-world problems typically have inaccurate data, it is of practical and theoretical interest to know the behavior of error bounds under data perturbations. For systems of linear inequalities, this question has been studied by Luo and Tseng [39] and Azé and Corvellec [5]. Subsequently Deng [16] studied systems of a finite number of convex inequalities. In 2005, Zheng and $\mathrm{Ng}$ [53] considered the stability of error bounds for systems of conic linear inequalities in a general Banach space. In 2010, Ngai, Kruger and Théra [42] studied the stability of error bounds for semi-infinite convex constraint systems in a Euclidean space and established subdifferential characterizations of the stability under small perturbations. The infinite dimensional extensions were considered by Kruger, Ngai and Théra in [35]. In 2012, by relaxing the convexity assumption, Zheng and Wei [56] discussed the stability of error bounds for quasi-subsmooth (not necessarily convex) inequalities in a general Banach space and provided Clarke subdifferential characterizations of the stability of error bounds. In 2018, Kruger, López and Théra [33] extended the development in [35, 42] and characterized the stability of error bounds for convex inequalities in the Banach space setting. From the viewpoint of infinite dimensional Banach spaces, results on the stability of error bounds in $[33,35,42,56]$ are dual conditions, and it is a pretty natural idea to study this issue not involving the dual space since information on the dual space may be missing. Inspired by this observation, we study characterizations of stability of local and global error bounds of a single convex inequality and semi-infinite convex constraint systems via directional derivatives. For a single convex inequality, we prove that the stability of local error bounds under small perturbations holds if and only if the minimum of the directional derivative at a reference point over the unit sphere is non-zero, and the stability of global error bounds is proved to be equivalent to the strictly positive infimum of the directional derivatives, at all points in the boundary of the solution set, over the unit sphere as well as some mild constraint qualification. When these results are applied to semi-infinite convex constraint systems, characterizations of the stability of local and global error bounds under small perturbations are also provided. Particularly such stability of error bounds is proved to only require that all component functions in semi-infinite convex constraint systems have the same linear perturbation. Our work demonstrates that verifying the stability of error bounds for convex inequality constraint systems is, to some degree, equivalent to solving optimization/minimization problems (defined by directional derivatives) over the unit sphere.

The paper is organized as follows. In Section 2, we give some definitions and preliminary results. Section 3 is devoted to the study on stability of error bounds for a single convex inequality. In terms of directional derivatives, we provide characterizations of local and global error bounds for a single convex inequality under small perturbations(see Theorem 5 and Theorem 10). When these results are applied to the semi-infinite convex constraint systems in Section 4, the stability of local and global error bounds can be obtained (see Theorem 13 and Theorem 15). Conclusions of this paper are given in Section 5.

\section{Preliminaries}

In what follows we consider the Euclidean space $\mathbb{R}^{m}$ equipped with the norm $\|\cdot\|:=\sqrt{\langle\cdot, \cdot\rangle}$. We denote by $\mathbf{B}^{m}$ the closed unit ball of $\mathbb{R}^{m}$ and following the standard notation by $\Gamma_{0}\left(\mathbb{R}^{m}\right)$ the set of extended-real-valued lower semicontinuous convex functions $f: \mathbb{R}^{m} \rightarrow \mathbb{R} \cup\{+\infty\}$ which are supposed to be proper, that is such that $\operatorname{dom}(f):=\left\{x \in \mathbb{R}^{m}: f(x)<+\infty\right\}$ is nonempty.

For a subset $D$ of $\mathbb{R}^{m}$, we denote by $d(x, D)$ the distance from $x$ to $D$ which is defined by

$$
d(x, D):=\inf \{\|x-y\|: y \in D\},
$$

where we use the convention $\inf \emptyset=+\infty$. We denote by $\operatorname{bdry}(D)$ and $\operatorname{int}(D)$ the boundary and the interior of $D$, respectively. 
Let $f \in \Gamma_{0}\left(\mathbb{R}^{m}\right)$ and $\bar{x} \in \operatorname{dom}(f)$. For any $h \in \mathbb{R}^{m}$, we recall that the directional derivative $f^{\prime}(\bar{x}, h)$ of $f$ at $\bar{x}$ along the direction $h$ is defined as

$$
f^{\prime}(\bar{x}, h):=\lim _{t \rightarrow 0^{+}} \frac{f(\bar{x}+t h)-f(\bar{x})}{t} .
$$

It is known from $[46,50]$ that the function

$$
t \mapsto \frac{f(\bar{x}+t h)-f(\bar{x})}{t}
$$

is nonincreasing as $t \rightarrow 0^{+}$and thus

$$
f^{\prime}(\bar{x}, h)=\inf _{t>0} \frac{f(\bar{x}+t h)-f(\bar{x})}{t} .
$$

We denote by $\partial f(\bar{x})$ the subdifferential of $f$ at $\bar{x}$ which is defined by

$$
\partial f(\bar{x}):=\left\{x^{*} \in \mathbb{R}^{m}:\left\langle x^{*}, x-\bar{x}\right\rangle \leq f(x)-f(\bar{x}) \text { for all } x \in \mathbb{R}^{m}\right\} .
$$

It is known from $[46,50]$ that if $\partial f(\bar{x})$ is nonempty, one has

$$
\partial f(\bar{x})=\left\{x^{*} \in \mathbb{R}^{m}:\left\langle x^{*}, h\right\rangle \leq f^{\prime}(\bar{x}, h) \text { for all } h \in \mathbb{R}^{m}\right\}
$$

and

$$
f^{\prime}(\bar{x}, h)=\sup \left\{\left\langle x^{*}, h\right\rangle: x^{*} \in \partial f(\bar{x})\right\},
$$

where we use the convention $\sup \emptyset=-\infty$.

We conclude this section with the following lemma which is used in our analysis.

- Lemma 1. Let $f \in \Gamma_{0}\left(\mathbb{R}^{m}\right)$ and $\bar{x} \in \operatorname{dom}(f)$ be such that $\inf _{\|h\|=1} f^{\prime}(\bar{x}, h)<0$. Then

$$
-\inf _{\|h\|=1} f^{\prime}(\bar{x}, h)=d(0, \partial f(\bar{x})) .
$$

Proof. If $\inf _{\|h\|=1} f^{\prime}(\bar{x}, h)=-\infty$, then one has $\partial f(\bar{x})=\emptyset$ by (4) and thus (5) holds.

Next, we consider the case $\inf _{\|h\|=1} f^{\prime}(\bar{x}, h)>-\infty$. Note that $\inf _{\|h\|=1} f^{\prime}(\bar{x}, h)<0$ and thus $0 \notin \partial f(\bar{x})$. Let $r:=d(0, \partial f(\bar{x}))$. By the separation theorem, there exists $h_{0} \in \mathbb{R}^{m}$ with $\left\|h_{0}\right\|=1$ such that

$$
\sup _{x^{*} \in \partial f(\bar{x})}\left\langle x^{*}, h_{0}\right\rangle+r=0
$$

and thus by (4), one has

$$
\inf _{\|h\|=1} f^{\prime}(\bar{x}, h) \leq f^{\prime}\left(\bar{x}, h_{0}\right)=-r .
$$

For any $\varepsilon>0$, we can select $u_{\varepsilon}^{*} \in(r+\varepsilon) \mathbf{B}^{m} \cap \partial f(\bar{x})$. Then for any $h \in \mathbb{R}^{m}$ with $\|h\|=1$, one has

$$
f^{\prime}(\bar{x}, h) \geq\left\langle u_{\varepsilon}^{*}, h\right\rangle \geq-(r+\varepsilon)
$$

and consequently

$$
\inf _{\|h\|=1} f^{\prime}(\bar{x}, h) \geq-(r+\varepsilon) .
$$

By letting $\varepsilon \rightarrow 0^{+}$, it follows from (6) that (5) holds. The proof is complete.

\section{Stability of Error Bounds for A Single Convex Inequality}

In this section, we mainly study local and global error bounds for a single convex inequality, and provide characterizations of stability (in terms of directional derivatives) of error bounds. We first recall the definition of error bounds for a single convex inequality. 
For a given $f \in \Gamma_{0}\left(\mathbb{R}^{m}\right)$, we consider the set of solutions of a single convex inequality:

$$
S_{f}:=\left\{x \in \mathbb{R}^{m}: f(x) \leq 0\right\} .
$$

Recall that convex inequality (7) is said to have a global error bound if there exists a constant $\tau \in(0,+\infty)$ such that

$$
d\left(x, S_{f}\right) \leq \tau[f(x)]_{+} \quad \forall x \in \mathbb{R}^{m},
$$

where $[f(x)]_{+}:=\max \{f(x), 0\}$. We denote by $\tau_{\min }(f):=\inf \{\tau>0:$ (8) holds $\}$ the global error bound modulus of $S_{f}$.

For $\bar{x} \in \operatorname{bdry}\left(S_{f}\right)$, convex inequality (7) is said to have a local error bound at $\bar{x}$ if there exist constants $\tau, \delta \in(0,+\infty)$ such that

$$
d\left(x, S_{f}\right) \leq \tau[f(x)]_{+} \quad \forall x \in B(\bar{x}, \delta) .
$$

We denote by $\tau_{\min }(f, \bar{x}):=\inf \{\tau>0:$ there exists $\delta>0$ such that (9) holds $\}$ the local error bound modulus of $S_{f}$ at $\bar{x}$.

The following theorem gives characterizations of global and local error bounds. We refer the readers to [5] for more details. This result is needed in the sequel.

- Theorem 2. Let $f \in \Gamma_{0}\left(\mathbb{R}^{m}\right)$. Then

i. $S_{f}$ has a global error bound if and only if

$\eta(f):=\inf \left\{d(0, \partial f(x)): x \in \mathbb{R}^{m}, f(x)>0\right\}>0$.

More precisely, $\tau_{\min }(f)=[\eta(f)]^{-1}$.

ii. $S_{f}$ has a local error bound at $\bar{x} \in \operatorname{bdry}\left(S_{f}\right)$ if and only if

$\eta(f, \bar{x}):=\liminf _{x \rightarrow \bar{x}, f(x)>0} d(0, \partial f(x))>0$.

More precisely, $\tau_{\min }(f, \bar{x})=[\eta(f, \bar{x})]^{-1}$.

iii. The following equality holds:

$$
\tau_{\min }(f)=\sup _{\bar{x} \in \text { bdry } S_{f}} \tau_{\min }(f, \bar{x})
$$

For a mapping $\phi: X \rightarrow Y$ between two normed linear spaces $X, Y$, we denote by $\operatorname{Lip}(\phi)$ the Lipschitz constant which is defined by

$$
\operatorname{Lip}(\phi):=\sup _{u, v \in X, u \neq v} \frac{\|\phi(u)-\phi(v)\|}{\|u-v\|} .
$$

\subsection{Stability of Local Error bounds}

In this subsection, we mainly study local error bounds for a single convex inequality and aim to provide equivalent criterion for the stability of local error bounds for convex inequality (7). We first give a sufficient condition for the local error bound of convex inequality (7).

- Proposition 3. Let $f \in \Gamma_{0}\left(\mathbb{R}^{m}\right)$ and $\bar{x} \in S_{f}$ such that $\inf _{\|h\|=1} f^{\prime}(\bar{x}, h) \neq 0$. Then convex inequality (7) has a local error bound at $\bar{x}$ and moreover

$$
\tau_{\min }(f, \bar{x}) \leq \frac{1}{\left|\inf _{\|h\|=1} f^{\prime}(\bar{x}, h)\right|} .
$$

Proof. Let $\beta(f, \bar{x}):=\inf _{\|h\|=1} f^{\prime}(\bar{x}, h)$. Suppose that $\beta(f, \bar{x})>0$. Then for any $x \neq \bar{x}$, by (2), one can verify that

$$
\begin{aligned}
f(x)-f(\bar{x}) & =f\left(\bar{x}+\|x-\bar{x}\| \frac{x-\bar{x}}{\|x-\bar{x}\|}\right)-f(\bar{x}) \\
& \geq f^{\prime}\left(\bar{x}, \frac{x-\bar{x}}{\|x-\bar{x}\|}\right)\|x-\bar{x}\| \\
& \geq \beta(f, \bar{x})\|x-\bar{x}\| \geq \beta(f, \bar{x}) d\left(x, S_{f}\right) .
\end{aligned}
$$


This means that $\tau_{\min }(f, \bar{x}) \leq[\beta(f, \bar{x})]^{-1}$.

Suppose that $\beta(f, \bar{x})<0$. Then Lemma 1 implies that $d(0, \partial f(\bar{x}))=-\beta(f, \bar{x})$ and by virtue of Theorem 2 , one has

$$
\tau(f, \bar{x}) \leq \frac{1}{-\beta(f, \bar{x})} .
$$

Hence (10) holds. The proof is complete.

Remark 4. Close analysis of the proof of Proposition 3 shows that the solution set $S_{f}$ will reduce to the singleton $\{\bar{x}\}$ if $\inf _{\|h\|=1} f^{\prime}(\bar{x}, h)>0$ and $f(\bar{x})=0$, which means that $\bar{x}$ is the sharp (or strong) minimizer of $f$. Further, it should be noted that the condition $\inf _{\|h\|=1} f^{\prime}(\bar{x}, h) \neq 0$ is only sufficient for the existence of a local error bound of $(7)$. Indeed, let $f(x) \equiv 0$ for all $x \in \mathbb{R}$. Then $S_{f}=\mathbb{R}$ has a global error bound, while $\inf _{\|h\|=1} f^{\prime}(\bar{x}, h)=0$ for all $\bar{x} \in \mathbb{R}$.

The following theorem shows that the condition $\inf _{\|h\|=1} f^{\prime}(\bar{x}, h) \neq 0$ can be used to give characterizations of stability of the local error bound for the convex inequality (7). For the sake of completeness, we provide a self-contained proof of this theorem.

- Theorem 5. Let $f \in \Gamma_{0}\left(\mathbb{R}^{m}\right)$ and $\bar{x} \in \mathbb{R}^{m}$ be such that $f(\bar{x})=0$. Then the following statements are equivalent: i. $\inf _{\|h\|=1} f^{\prime}(\bar{x}, h) \neq 0$;

ii. There exist constants $c, \varepsilon>0$ such that for all $g \in \Gamma_{0}\left(\mathbb{R}^{m}\right)$ satisfying $\bar{x} \in S_{g}$ and

$$
\limsup _{x \rightarrow \bar{x}} \frac{|(f(x)-g(x))-(f(\bar{x})-g(\bar{x}))|}{\|x-\bar{x}\|} \leq \varepsilon,
$$

one has $\tau_{\min }(g, \bar{x}) \leq c$;

iii. There exist constants $c, \varepsilon>0$ such that for all $u^{*} \in \mathbb{R}^{m}$ with $\left\|u^{*}\right\| \leq 1$, one has $\tau_{\min }\left(g_{u^{*}, \varepsilon}, \bar{x}\right) \leq c$, where $g_{u^{*}, \varepsilon}(x):=f(x)+\varepsilon\left\langle u^{*}, x-\bar{x}\right\rangle$ for all $x \in \mathbb{R}^{m}$.

Proof. Let $\beta(f, \bar{x}):=\inf _{\|h\|=1} f^{\prime}(\bar{x}, h)$.

Let us first prove $\mathrm{i} \Rightarrow$ ii. Take any $\varepsilon>0$ such that $\varepsilon<|\beta(f, \bar{x})|$ and let $c:=(|\beta(f, \bar{x})|-\varepsilon)^{-1}$. For any $g \in \Gamma_{0}\left(\mathbb{R}^{m}\right)$ such that $\bar{x} \in S_{g}$ and (11) holds. If $\beta(f, \bar{x})>0$, then for any $h \in \mathbb{R}^{m}$, one has

$$
g^{\prime}(\bar{x}, h) \geq f^{\prime}(\bar{x}, h)-\varepsilon,
$$

and thus

$$
\inf _{\|h\|=1} g^{\prime}(\bar{x}, h) \geq \inf _{\|h\|=1} f^{\prime}(\bar{x}, h)-\varepsilon \geq \beta(f, \bar{x})-\varepsilon .
$$

This and Proposition 3 imply that $\tau_{\min }(g, \bar{x}) \leq[\beta(f, \bar{x})-\varepsilon]^{-1}=c$.

If $\beta(f, \bar{x})<0$, then for any $h \in \mathbb{R}^{m}$, one has

$$
g^{\prime}(\bar{x}, h) \leq f^{\prime}(\bar{x}, h)+\varepsilon,
$$

and thus

$$
\inf _{\|h\|=1} g^{\prime}(\bar{x}, h) \leq \inf _{\|h\|=1} f^{\prime}(\bar{x}, h)+\varepsilon \leq \beta(f, \bar{x})+\varepsilon .
$$

By using Proposition 3 again, one yields that $\tau_{\min }(g, \bar{x}) \leq[-\beta(f, \bar{x})-\varepsilon]^{-1}=c$. Hence ii holds.

Note that the implication ii $\Rightarrow$ iii is clear and it remains to prove iii $\Rightarrow$ i.

Let $\varepsilon>0$. Suppose on the contrary that there exists a sequence $\left\{h_{k}\right\}$ in $\mathbb{R}^{m}$ with $\left\|h_{k}\right\|=1$ such that

$$
\alpha_{k}:=f^{\prime}\left(\bar{x}, h_{k}\right) \rightarrow 0 .
$$

Without loss of generality, we can assume that $\left|\alpha_{k}\right|<\varepsilon$ for all $k$ (considering sufficiently large $k$ if necessary) and consider the function $g_{\varepsilon}(x):=f(x)+\varepsilon\left\langle h_{k}, x-\bar{x}\right\rangle$ for all $x \in \mathbb{R}^{m}$. From $\beta(f, \bar{x})=0$, one can verify that $f(x) \geq f(\bar{x})$ for any $x \neq \bar{x}$. By the definition of directional derivative, there exists a sequence $\left\{\delta_{k}\right\}$ decreasing to 0 such that

$$
f\left(\bar{x}+\delta_{k} h_{k}\right)<f(\bar{x})+\left(\varepsilon+\alpha_{k}\right) \delta_{k}=\inf _{x \in \mathbb{R}^{m}} f(x)+\left(\varepsilon+\alpha_{k}\right) \delta_{k} .
$$


By virtue of the Ekeland variational principle, we can select $z_{k} \in \mathbb{R}^{m}$ such that $\left\|z_{k}-\left(\bar{x}+\delta_{k} h_{k}\right)\right\|<\frac{\delta_{k}}{2}, f\left(z_{k}\right) \leq$ $f\left(\bar{x}+\delta_{k} h_{k}\right)$ and

$$
f(x)+2\left(\varepsilon+\alpha_{k}\right)\left\|x-z_{k}\right\|>f\left(z_{k}\right), \forall x \neq z_{k} .
$$

This implies that $z_{k} \rightarrow \bar{x}, g_{\varepsilon}(\bar{x})=f(\bar{x})=0$ and

$$
\begin{aligned}
g_{\varepsilon}\left(z_{k}\right) & =f\left(z_{k}\right)+\varepsilon\left\langle h_{k}, z_{k}-\bar{x}\right\rangle \\
& \geq f(\bar{x})+\varepsilon\left\langle h_{k}, z_{k}-\bar{x}\right\rangle \\
& =\varepsilon\left\langle h_{k}, z_{k}-\bar{x}-\delta_{k} h_{k}\right\rangle+\varepsilon \delta_{k} \\
& >\varepsilon \delta_{k}-\frac{1}{2} \varepsilon \delta_{k}=\frac{1}{2} \varepsilon \delta_{k}>0 .
\end{aligned}
$$

We claim that

$$
\inf _{\|h\|=1} g_{\varepsilon}^{\prime}\left(z_{k}, h\right)<0 .
$$

(Otherwise, $\inf _{\|h\|=1} g_{\varepsilon}^{\prime}\left(z_{k}, h\right) \geq 0$ and then one has $g_{\varepsilon}\left(z_{k}\right)=\inf _{x \in \mathbb{R}^{m}} g_{\varepsilon}(x)$, which contradicts $\left.g_{\varepsilon}(\bar{x})=0\right)$.

For any $h \in \mathbb{R}^{m}$ with $\|h\|=1$ and any $t>0$, by (13), one has

$$
\frac{g_{\varepsilon}\left(z_{k}+t h\right)-g_{\varepsilon}\left(z_{k}\right)}{t}=\frac{f\left(z_{k}+t h\right)-f\left(z_{k}\right)}{t}+\varepsilon\left\langle h_{k}, h\right\rangle \geq-2\left(\varepsilon+\alpha_{k}\right)\|h\|-\varepsilon=-5 \varepsilon
$$

and consequently

$$
0 \geq \inf _{\|h\|=1} g_{\varepsilon}^{\prime}\left(z_{k}, h\right) \geq-5 \varepsilon .
$$

Thanks to Lemma 1 and Proposition 3, one can obtain that $\tau_{\min }\left(g_{\varepsilon}, \bar{x}\right) \geq \frac{1}{5 \varepsilon}$, which contradicts iii as $\varepsilon$ is arbitrary. The proof is complete.

Remark 6. (a). From [33, 42], the condition (11) means that $g$ is an $\varepsilon$-perturbation of $f$ near $\bar{x}$, and the condition $\inf _{\|h\|=1} f^{\prime}(\bar{x}, h) \neq 0$ is proved to be equivalent to the stability of this $\varepsilon$-perturbation of local error bounds. Further, it has been shown in Theorem 5 that the stability of such $\varepsilon$-perturbation is essentially equivalent to that of $\varepsilon$-linear perturbation.

(b). Theorem 5 can be regarded as the equivalent version of [42, Theorem 2] since one can prove that

$$
\inf _{\|h\|=1} f^{\prime}(\bar{x}, h) \neq 0 \Longleftrightarrow 0 \notin \operatorname{bdry}(\partial f(\bar{x})) .
$$

Indeed, suppose that $0 \notin \operatorname{bdry}(\partial f(\bar{x}))$. For the case that $0 \in \operatorname{int}(\partial f(\bar{x}))$, there is $r>0$ such that $r \mathbf{B}^{m} \subseteq \partial f(\bar{x})$. This and (4) imply that

$$
\inf _{\|h\|=1} f^{\prime}(\bar{x}, h) \geq r>0 .
$$

For the case that $0 \notin \partial f(\bar{x})$, by the separation theorem, there exists $h_{0} \in \mathbb{R}^{m}$ with $\left\|h_{0}\right\|=1$ such that

$$
0>\sup \left\{\left\langle x^{*}, h_{0}\right\rangle: x^{*} \in \partial f(\bar{x})\right\}=f^{\prime}\left(\bar{x}, h_{0}\right)
$$

and consequently

$$
\inf _{\|h\|=1} f^{\prime}(\bar{x}, h) \leq f^{\prime}\left(\bar{x}, h_{0}\right)<0 .
$$

On the other hand, if $0 \in \operatorname{bdry}(\partial f(\bar{x}))$, then $\inf _{\|h\|=1} f^{\prime}(\bar{x}, h) \geq 0$ and for any $\varepsilon>0$, we can select $u_{\varepsilon}^{*} \in \varepsilon \mathbf{B}^{m} \backslash \partial f(\bar{x})$ and $x_{\varepsilon} \neq \bar{x}$ such that

$$
\left\langle u_{\varepsilon}^{*}, x_{\varepsilon}-\bar{x}\right\rangle>f\left(x_{\varepsilon}\right)-f(\bar{x}) .
$$

By (2), for any $t \in(0,1)$, one has

$$
\frac{f\left(\bar{x}+t\left(x_{\varepsilon}-\bar{x}\right)\right)-f(\bar{x})}{t} \leq f\left(x_{\varepsilon}\right)-f(\bar{x})<\left\langle u_{\varepsilon}^{*}, x_{\varepsilon}-\bar{x}\right\rangle
$$

and thus

$$
f^{\prime}\left(\bar{x}, \frac{x_{\varepsilon}-\bar{x}}{\left\|x_{\varepsilon}-\bar{x}\right\|}\right) \leq\left\langle u_{\varepsilon}^{*}, \frac{x_{\varepsilon}-\bar{x}}{\left\|x_{\varepsilon}-\bar{x}\right\|}\right\rangle \leq \varepsilon .
$$

This means that $\inf _{\|h\|=1} f^{\prime}(\bar{x}, h) \leq \varepsilon \rightarrow 0^{+}$and so $\inf _{\|h\|=1} f^{\prime}(\bar{x}, h)=0$. 


\subsection{Stability of Global Error Bounds}

This subsection is devoted to the study of stability of global error bounds for a single convex inequality, and the aim is to give sufficient and/or necessary conditions for the stability via directional derivatives. The following theorem gives a criterion for the stability of global error bounds.

- Theorem 7. Let $f \in \Gamma_{0}\left(\mathbb{R}^{m}\right)$ be such that $\operatorname{bdry}\left(S_{f}\right) \subseteq f^{-1}(0)$. Consider the following statements:

i. There exists $\tau \in(0,+\infty)$ such that

$$
\inf \left\{\left|\inf _{\|h\|=1} f^{\prime}(\bar{x}, h)\right|: \bar{x} \in \operatorname{bdry}\left(S_{f}\right)\right\}>\tau \text {. }
$$

ii. There exist constants $c, \varepsilon \in(0,+\infty)$ such that for all $g \in \Gamma_{0}\left(\mathbb{R}^{m}\right)$ satisfying

$S_{f} \subseteq S_{g}$ and $\operatorname{Lip}(f-g)<\varepsilon$,

one has $\tau_{\min }(g) \leq c$.

iii. There exist constants $c, \varepsilon \in(0,+\infty)$ such that for all $g \in \Gamma_{0}\left(\mathbb{R}^{m}\right)$ satisfying

$\operatorname{bdry}\left(S_{f}\right) \cap g^{-1}(0) \neq \emptyset$ and $\operatorname{Lip}(f-g)<\varepsilon$,

one has $\tau_{\min }(g) \leq c$.

Then iii $\Rightarrow$ i $\Rightarrow$ ii.

Proof. $\mathbf{i} \Rightarrow$ ii. If there is some $\bar{x} \in \operatorname{bdry}\left(S_{f}\right)$ such that $\inf _{\|h\|=1} f^{\prime}(\bar{x}, h)>0$, then the implication follows by Remark 4 and the proof of Theorem 5 .

We next consider the case $\inf _{\|h\|=1} f^{\prime}(\bar{x}, h) \leq 0$ for all $\bar{x} \in \operatorname{bdry}\left(S_{f}\right)$. By virtue of (15) and Theorem 2, one can verify that $S_{f}$ has a global error bound with the constant $\frac{1}{\tau}$; that is,

$$
d\left(x, S_{f}\right) \leq \frac{1}{\tau}[f(x)]_{+}, \quad \forall x \in \mathbb{R}^{m}
$$

Take any $\varepsilon \in(0, \tau)$. Suppose that $g \in \Gamma_{0}\left(\mathbb{R}^{m}\right)$ satisfies (16). Let $x \in \mathbb{R}^{m}$ be such that $g(x)>0$. Then $f(x)>0$ as $S_{f} \subseteq S_{g}$. We claim that

$$
\inf _{\|h\|=1} f^{\prime}(x, h) \leq-\tau \text {. }
$$

Granting this, by $\operatorname{Lip}(f-g)<\varepsilon$ in $(16)$, one can prove that

$$
\inf _{\|h\|=1} g^{\prime}(x, h) \leq \inf _{\|h\|=1} f^{\prime}(x, h)+\varepsilon \leq-(\tau-\varepsilon) .
$$

This and Theorem 2 imply that $\tau_{\min }(g) \leq(\tau-\varepsilon)^{-1}$.

We next prove the claim (19). Take $z \in \operatorname{bdry}\left(S_{f}\right)$ such that $\|x-z\|=d\left(x, S_{f}\right)$ and (18) implies that

$$
f(x) \geq \tau d\left(x, S_{f}\right)=\tau\|x-z\| .
$$

Then for any $t \in(0,1)$, one has

$$
f(x+t(z-x)) \leq t f(z)+(1-t) f(x)
$$

and thus

$$
\frac{f(x+t(z-x))-f(x)}{t} \leq-f(x) \leq-\tau\|x-z\| .
$$

This means that

$$
\inf _{\|h\|=1} f^{\prime}(\bar{x}, h) \leq f^{\prime}\left(x, \frac{z-x}{\|x-z\|}\right) \leq-\tau
$$

Hence (19) holds. 
iii $\Rightarrow \mathbf{i}$. Suppose that there exists a sequence $\left\{x_{k}\right\} \subseteq \operatorname{bdry}\left(S_{f}\right)$ such that

$$
\alpha_{k}:=\inf _{\|h\|=1} f^{\prime}\left(x_{k}, h\right) \rightarrow 0^{-}(\text {as } k \rightarrow \infty) .
$$

Let $\varepsilon>0$ be arbitrary and $k$ be sufficiently large such that

$$
\frac{3}{2} \alpha_{k}+\frac{\varepsilon}{2}>0
$$

Note that for any $x \neq x_{k}$, one has

$$
\frac{f(x)-f\left(x_{k}\right)}{\left\|x-x_{k}\right\|}=\frac{f\left(x_{k}+\left\|x-x_{k}\right\| \cdot \frac{x-x_{k}}{\left\|x-x_{k}\right\|}\right)-f\left(x_{k}\right)}{\left\|x-x_{k}\right\|} \geq f^{\prime}\left(x_{k}, \frac{x-x_{k}}{\left\|x-x_{k}\right\|}\right) \geq \alpha_{k}
$$

and thus

$$
f(x)-\alpha_{k}\left\|x-x_{k}\right\| \geq f\left(x_{k}\right), \quad \forall x \in \mathbb{R}^{m} .
$$

Choose $h_{k} \in \mathbb{R}^{m}$ with $\left\|h_{k}\right\|=1$ such that

$$
f^{\prime}\left(x_{k}, h_{k}\right)<\alpha_{k}+\frac{\varepsilon}{2} \text {. }
$$

Then we can take $r_{k} \rightarrow 0^{+}$(as $\left.k \rightarrow \infty\right)$ such that

$$
f\left(x_{k}+r_{k} h_{k}\right)<f\left(x_{k}\right)+\left(\alpha_{k}+\varepsilon\right) r_{k} .
$$

This and (21) imply that

$$
f\left(x_{k}+r_{k} h_{k}\right)-\alpha_{k}\left\|x_{k}+r_{k} h_{k}-x_{k}\right\|<\inf _{x \in \mathbb{R}^{m}}\left(f(x)-\alpha_{k}\left\|x-x_{k}\right\|\right)+\varepsilon r_{k} .
$$

Applying Ekeland variational principle, we can select $y_{k} \in \mathbb{R}^{m}$ such that

$$
\left\|y_{k}-\left(x_{k}+r_{k} h_{k}\right)\right\|<\frac{r_{k}}{2}, f\left(y_{k}\right)-\alpha_{k}\left\|y_{k}-x_{k}\right\| \leq f\left(x_{k}+r_{k} h_{k}\right)-\alpha_{k} r_{k},
$$

and

$$
f(x)-\alpha_{k}\left\|x-x_{k}\right\|+2 \varepsilon\left\|x-y_{k}\right\|>f\left(y_{k}\right)-\alpha_{k}\left\|y_{k}-x_{k}\right\|, \quad \forall x \neq y_{k} .
$$

This implies that

$$
\left\|y_{k}-x_{k}\right\|>r_{k}-\frac{r_{k}}{2}=\frac{r_{k}}{2} \text { and }\left\|y_{k}-x_{k}\right\|<r_{k}+\frac{r_{k}}{2}=\frac{3}{2} r_{k}
$$

and thus $y_{k} \neq x_{k}$. Let us consider a function $g_{\varepsilon} \in \Gamma_{0}\left(\mathbb{R}^{m}\right)$ defined by

$$
g_{\varepsilon}(x):=f(x)+\varepsilon\left\langle h_{k}, x-x_{k}\right\rangle \text { for all } x \in \mathbb{R}^{m} .
$$

By virtue of (20), (21), (22) and (25), one has

$$
\begin{aligned}
g_{\varepsilon}\left(y_{k}\right)=f\left(y_{k}\right)+\varepsilon\left\langle h_{k}, y_{k}-x_{k}\right\rangle & =f\left(y_{k}\right)+\varepsilon\left\langle h_{k}, y_{k}-\left(x_{k}+r_{k} h_{k}\right)\right\rangle+\varepsilon r_{k} \\
& \geq \alpha_{k}\left\|y_{k}-x_{k}\right\|-\varepsilon\left\|y_{k}-\left(x_{k}+r_{k} h_{k}\right)\right\|+\varepsilon r_{k} \\
& \geq \alpha_{k} \cdot \frac{3}{2} r_{k}+\frac{\varepsilon}{2} r_{k}>0 .
\end{aligned}
$$

If $\inf _{\|h\|=1} g_{\varepsilon}^{\prime}\left(y_{k}, h\right) \geq 0$, then for any $x \neq y_{k}$, one has

$$
g_{\varepsilon}(x)-g_{\varepsilon}\left(y_{k}\right) \geq g_{\varepsilon}^{\prime}\left(y_{k}, \frac{x-y_{k}}{\left\|x-y_{k}\right\|}\right)\left\|x-y_{k}\right\| \geq \inf _{\|h\|=1} g_{\varepsilon}^{\prime}\left(y_{k}, h\right)\left\|x-y_{k}\right\| \geq 0 .
$$

This and $g_{\varepsilon}\left(y_{k}\right)>0$ imply that $S_{g_{\varepsilon}}=\emptyset$, and thus $\tau_{\min }\left(g_{\varepsilon}\right)=+\infty$, which contradicts iii. 
Next, we consider the case $\inf _{\|h\|=1} g_{\varepsilon}^{\prime}\left(y_{k}, h\right)<0$. For any $h \in \mathbb{R}^{m}$ with $\|h\|=1$ and $t>0$, by (25), one has

$$
\begin{aligned}
\frac{g_{\varepsilon}\left(y_{k}+t h\right)-g_{\varepsilon}\left(y_{k}\right)}{t} & =\frac{f\left(y_{k}+t h\right)-f\left(y_{k}\right)}{t}+\varepsilon\left\langle h_{k}, h\right\rangle \\
& \geq \frac{1}{t}\left(\alpha_{k}\left\|y_{k}+t h-x_{k}\right\|-\alpha_{k}\left\|y_{k}-x_{k}\right\|-2 \varepsilon\left\|y_{k}+t h-y_{k}\right\|\right)+\varepsilon\left\langle h_{k}, h\right\rangle \\
& \geq \alpha_{k}-2 \varepsilon-\varepsilon
\end{aligned}
$$

and consequently

$$
0>\inf _{\|h\|=1} g_{\varepsilon}^{\prime}\left(y_{k}, h\right) \geq \alpha_{k}-2 \varepsilon-\varepsilon \geq-4 \varepsilon .
$$

Thanks to Lemma 1 and Theorem 2, we obtain $\tau_{\min }\left(g_{\varepsilon}\right) \geq \frac{1}{4 \varepsilon}$, which contradicts iii as $\varepsilon$ is arbitrary. The proof is complete.

Remark 8. (a). Compared with [42, Theorem 7] in which a subdifferential characterization of stability of global error bounds was established with the aid of the so-called asymptotic qualification condition, Theorem 7 studies the stability of global error bounds via directional derivatives without additional hypothesis. It is known from Theorem 7 that the condition (15) is sufficient for the stability of global error bounds as said in Theorem 7.ii, and is necessary for the stability as in Theorem 7.iii.

(b). It should be noted that the condition (15) is not sufficient for the stability of global error bounds as in Theorem 7.iii, and the assumption $S_{f} \subseteq S_{g}$ for the stability as said in Theorem 7.ii is crucial. To see this, let us consider the following example:

\section{Example 9.}

Let $f(x):=e^{x}-1$ for all $x \in \mathbb{R}$. Then $S_{f}=(-\infty, 0]$, $\operatorname{bdry}\left(S_{f}\right)=\{0\}$ and $\left|\inf _{|h|=1} f^{\prime}(0, h)\right|=1>0$. However, for any $\varepsilon \in(0,+\infty)$, let us consider the function $g_{\varepsilon}(x):=f(x)-\varepsilon x$ for all $x \in \mathbb{R}$. Then one can verify that $g_{\varepsilon}$ has two different zero points which are denoted by $x_{1}:=\bar{x}<0$ and $x_{2}:=0$ and $S_{g_{\varepsilon}}=[\bar{x}, 0]$. Thus $S_{f} \nsubseteq S_{g_{\varepsilon}}$ and for any $x<\bar{x}$, one has

$$
\frac{d\left(x, S_{g_{\varepsilon}}\right)}{g_{\varepsilon}(x)}=\frac{\bar{x}-x}{e^{x}-1-\varepsilon x} \rightarrow \frac{1}{\varepsilon} \text { as } x \rightarrow-\infty .
$$

This implies that

$$
\tau_{\min }\left(g_{\varepsilon}\right) \geq \frac{1}{2 \varepsilon},
$$

and consequently the global stability (for $f$ ) as said in Theorem 7.iii does not hold as $\varepsilon>0$ is arbitrary.

Further, a natural question arises from the above example:

Does there exist some type of stability of global error bounds that can be characterized by condition (15)?

We do not have an answer to this question. However, if the answer is affirmative, we conjecture that such global stability should be strictly stronger than that of Theorem 7.ii and weaker than that of Theorem 7.iii.

The following theorem gives characterizations of the stability of global error bounds for a convex inequality as said in Theorem 7.iii.

- Theorem 10. Let $f \in \Gamma_{0}\left(\mathbb{R}^{m}\right)$ be such that $\operatorname{bdry}\left(S_{f}\right) \subseteq f^{-1}(0)$. Then the following statements are equivalent:

i. There exists $\tau \in(0,+\infty)$ such that (15) holds and the following qualification condition is satisfied:

For any sequence $\left\{z_{k}\right\} \subseteq S_{f} \backslash \operatorname{bdry}\left(S_{f}\right)$, one has $\liminf _{k \rightarrow \infty}\left|\inf _{\|h\|=1} f^{\prime}\left(z_{k}, h\right)\right|>\tau$

if there is a sequence $\left\{x_{k}\right\} \subseteq \operatorname{bdry}\left(S_{f}\right)$ satisfying $\lim _{k \rightarrow \infty} \frac{f\left(z_{k}\right)-f\left(x_{k}\right)}{\left\|z_{k}-x_{k}\right\|}=0$.

ii. There exist constants $c, \varepsilon \in(0,+\infty)$ such that for all $g \in \Gamma_{0}\left(\mathbb{R}^{m}\right)$ satisfying $(17)$, one has $\tau_{\min }(g) \leq c$;

iii. There exist constants $c, \varepsilon>0$ such that for any $\bar{x} \in \operatorname{bdry}\left(S_{f}\right)$ and $u \in \mathbb{R}^{m}$ with $\|u\| \leq 1$, one has $\tau_{\min }\left(g_{u, \varepsilon}\right) \leq c$, where $g_{u, \varepsilon}(x):=f(x)+\varepsilon\langle u, x-\bar{x}\rangle$ for all $x \in \mathbb{R}^{m}$. 
Proof. Let us first prove i $\Rightarrow$ ii. Based on Remark 4 and the proof of Theorem 7, we only need to consider the case $\inf _{\|h\|=1} f^{\prime}(\bar{x}, h) \leq 0$ for all $\bar{x} \in \operatorname{bdry}\left(S_{f}\right)$. We first prove the following claim:

$\triangleright$ Claim. There exists $\varepsilon_{0}>0$ such that for all $x_{0} \in \operatorname{bdry}\left(S_{f}\right)$, one has

$$
\inf \left\{\left|\inf _{\|h\|=1} f^{\prime}\left(z_{0}, h\right)\right|: z_{0} \in \mathbb{R}^{m}, f\left(z_{0}\right) \geq-\varepsilon_{0}\left\|z_{0}-x_{0}\right\|\right\} \geq \tau .
$$

Proof of the claim. Suppose on the contrary that there exist $\varepsilon_{k} \rightarrow 0^{+}, x_{k} \in \operatorname{bdry}\left(S_{f}\right)$ and $z_{k} \in \mathbb{R}^{m}$ such that

$$
f\left(z_{k}\right) \geq-\varepsilon_{k}\left\|z_{k}-x_{k}\right\| \text { and }\left|\inf _{\|h\|=1} f^{\prime}\left(z_{k}, h\right)\right|<\tau \text { for all } k .
$$

Then $f\left(z_{k}\right) \leq 0$ for all $k$ (otherwise, similar to the proof of (19), one can prove that $\left|\inf _{\|h\|=1} f^{\prime}\left(z_{k}, h\right)\right|>\tau$, a contradiction). By (15), one has $z_{k} \in S_{f} \backslash \operatorname{bdry}\left(S_{f}\right)$ and it follows from (27) that

$$
0 \geq \frac{f\left(z_{k}\right)-f\left(x_{k}\right)}{\left\|z_{k}-x_{k}\right\|}=\frac{f\left(z_{k}\right)}{\left\|z_{k}-x_{k}\right\|} \geq-\varepsilon_{k} .
$$

This and the qualification condition in i imply that

$$
\liminf _{k \rightarrow \infty}\left|\inf _{\|h\|=1} f^{\prime}\left(z_{k}, h\right)\right|>\tau
$$

which contradicts (27). Hence the claim is proved.

Let $\varepsilon>0$ be such that $\varepsilon<\min \left\{\varepsilon_{0}, \tau\right\}$. Suppose that $g \in \Gamma_{0}\left(\mathbb{R}^{m}\right)$ satisfies (17). Take any $\bar{x} \in \operatorname{bdry}\left(S_{f}\right) \cap g^{-1}(0)$. Then for any $x \in \mathbb{R}^{m}$ with $g(x)>0$, one has

$$
f(x) \geq g(x)+(f(\bar{x})-g(\bar{x}))-\varepsilon\|x-\bar{x}\|>-\varepsilon\|x-\bar{x}\| .
$$

Using (26), one obtains

$$
\inf _{\|h\|=1} f^{\prime}(x, h)<-\tau
$$

and thus

$$
\inf _{\|h\|=1} g^{\prime}(x, h)<\inf _{\|h\|=1} f^{\prime}(x, h)+\varepsilon<-(\tau-\varepsilon) .
$$

By virtue of Lemma 1 and Theorem 2 we derive the inequality $\tau_{\min }(g) \leq \frac{1}{\tau-\varepsilon}$.

Note that ii $\Rightarrow$ iii follows immediately and it remains to prove iii $\Rightarrow$ i.

Suppose on the contrary that i does not hold. Based on iii $\Rightarrow \mathrm{i}$ in Theorem 7 , we only consider the case that there exist $z_{k} \in S_{f} \backslash \operatorname{bdry}\left(S_{f}\right)$ and $x_{k} \in \operatorname{bdry}\left(S_{f}\right)$ such that

$$
\lim _{k \rightarrow \infty} \frac{f\left(z_{k}\right)-f\left(x_{k}\right)}{\left\|z_{k}-x_{k}\right\|}=0 \text { and } \alpha_{k}:=\inf _{\|h\|=1} f^{\prime}\left(z_{k}, h\right) \rightarrow 0^{-} .
$$

Let $\varepsilon>0$ be arbitrary. Without loss of generality, we can assume that $\frac{z_{k}-x_{k}}{\left\|z_{k}-x_{k}\right\|} \rightarrow h_{0}$ (considering subsequence if necessary). Then $\left\|h_{0}\right\|=1$. Suppose that $k$ is sufficiently large such that

$$
\alpha_{k}+\varepsilon>0 \text { and } \frac{f\left(z_{k}\right)-f\left(x_{k}\right)}{\left\|z_{k}-x_{k}\right\|}+\varepsilon\left\langle h_{0}, \frac{z_{k}-x_{k}}{\left\|z_{k}-x_{k}\right\|}\right\rangle>0
$$

Let us consider a function $g_{h_{0}, \varepsilon} \in \Gamma_{0}\left(\mathbb{R}^{m}\right)$ defined by

$$
g_{h_{0}, \varepsilon}(x):=f(x)+\varepsilon\left\langle h_{0}, x-x_{k}\right\rangle \text { for all } x \in \mathbb{R}^{m} .
$$

Then $g_{h_{0}, \varepsilon}\left(z_{k}\right)=f\left(z_{k}\right)+\varepsilon\left\langle h_{0}, z_{k}-x_{k}\right\rangle>0$ by (29) and thus

$$
0>\inf _{\|h\|=1} g_{h_{0}, \varepsilon}^{\prime}\left(z_{k}, h\right) \geq \inf _{\|h\|=1} f^{\prime}\left(z_{k}, h\right)-\varepsilon=\alpha_{k}-\varepsilon>-2 \varepsilon .
$$

This together with Lemma 1 and Theorem 2 implies that $\tau_{\min }\left(g_{h_{0}, \varepsilon}\right) \geq \frac{1}{2 \varepsilon}$, which contradicts iii as $\varepsilon$ is arbitrary. The proof is complete. 
Remark 11. Note that condition (QC) is necessary for the stability of global error bounds. Consider Example 9 given in Remark 8 again. Let $f(x):=e^{x}-1$ for all $x \in \mathbb{R}$. Then the stability of global error bounds for $f$ as said in Theorem 7.iii does not hold. Further, for any $z_{k} \rightarrow-\infty$, one can verify that

$$
\left|\inf _{|h|=1} f^{\prime}\left(z_{k}, h\right)\right|=e^{z_{k}} \rightarrow 0 \text { as } k \rightarrow \infty,
$$

which means that (QC) (for $f$ ) fails.

\section{Stability of error bounds for Semi-infinite Convex Constraint Systems}

In this section, we study local and global error bounds for semi-infinite convex constraint systems, and mainly provide characterizations of stability of error bounds by directional derivatives. We first recall the definition of error bounds for semi-infinite convex constraint systems.

For semi-infinite convex constraint systems in $\mathbb{R}^{m}$, we mean the problem of finding $x \in \mathbb{R}^{m}$ satisfying:

$$
f_{i}(x) \leq 0 \text { for all } i \in I,
$$

where $I$ is a compact, possibly infinite, Hausdorff space, $f_{i}: \mathbb{R}^{m} \rightarrow \mathbb{R}, i \in I$, are given convex functions such that $i \mapsto f_{i}(x)$ is continuous on $I$ for each $x \in \mathbb{R}^{m}$. It is known from [50, Theorem 7.10] that in this case, $(i, x) \mapsto f_{i}(x)$ is continuous on $I \times \mathbb{R}^{m}$.

Let $F \in C\left(I \times \mathbb{R}^{m}, \mathbb{R}\right)$ be defined by $F(i, x):=f_{i}(x)$ for all $(i, x) \in I \times \mathbb{R}^{m}$. We denote the solution set of system (30) by

$$
S_{F}:=\left\{x \in \mathbb{R}^{m}: f_{i}(x) \leq 0 \text { for all } i \in I\right\} .
$$

For any $x \in \mathbb{R}^{m}$, we set

$$
f(x):=\max \left\{f_{i}(x): i \in I\right\} \text { and } I_{f}(x):=\left\{i \in I: f_{i}(x)=f(x)\right\} .
$$

Recall that system (30) is said to have a global error bound if there exists a constant $\tau \in(0,+\infty)$ such that

$$
d\left(x, S_{F}\right) \leq \tau[f(x)]_{+} \quad \forall x \in \mathbb{R}^{m} .
$$

We denote by $\tau_{\min }(F):=\inf \{\tau>0:(33)$ holds $\}$ the global error bound modulus of $S_{F}$.

For $\bar{x} \in \operatorname{bdry}\left(S_{F}\right)$, system (30) is said to have a local error bound at $\bar{x}$ if there exist constants $\tau, \delta \in(0,+\infty)$ such that

$$
d\left(x, S_{F}\right) \leq \tau[f(x)]_{+} \quad \forall x \in B(\bar{x}, \delta) .
$$

We denote by $\tau_{\min }(F, \bar{x}):=\inf \{\tau>0:$ there exists $\delta>0$ such that (34) holds $\}$ the local error bound modulus of $S_{F}$ at $\bar{x}$.

We first study stability of local error bounds for semi-infinite convex constraint system (30) and aim to provide characterizations of the stability of local error bounds for system (30). To this aim, we need the following proposition which is of independent interest.

- Proposition 12. Let $x \in \mathbb{R}^{m}$. Then for any $h \in \mathbb{R}^{m}$, one has

$$
f^{\prime}(x, h)=\max _{i \in I_{f}(x)} f_{i}^{\prime}(x, h) .
$$

Proof. Let $h \in \mathbb{R}^{m}$. Take any $i \in I_{f}(x)$. Then for any $t>0$, one has

$$
\frac{f_{i}(x+t h)-f_{i}(x)}{t} \leq \frac{f(x+t h)-f(x)}{t}
$$

and thus $f_{i}^{\prime}(x, h) \leq f^{\prime}(x, h)$. This implies that

$$
f^{\prime}(x, h) \geq \max _{i \in I_{f}(x)} f_{i}^{\prime}(x, h) .
$$

By virtue of (4), one has

$$
f^{\prime}(x, h)=\max _{x \in \partial f(x)}\left\langle x^{*}, h\right\rangle,
$$


and thus there is $z^{*} \in \partial f(x)$ such that

$$
f^{\prime}(x, h)=\left\langle z^{*}, h\right\rangle .
$$

Note that the subdifferential of the function $f$ at a point $x \in \mathbb{R}^{m}$ is given by (see Ioffe $\&$ Tikhomirov [24])

$$
\partial f(x)=\operatorname{co}\left(\bigcup_{i \in I_{f}(x)} \partial f_{i}(x)\right)
$$

where "co" denotes the convex hull of a set. Then by (37), there exist $\lambda_{1}, \ldots, \lambda_{N} \geq 0, i_{1}, \ldots, i_{N} \in I_{f}(x)$ and $z_{k}^{*} \in \partial f_{i_{k}}(x), k=1, \ldots, N$ such that

$$
\sum_{k=1}^{N} \lambda_{k}=1 \text { and } z^{*}=\sum_{k=1}^{N} \lambda_{k} z_{k}^{*} .
$$

This and (37) imply that

$$
f^{\prime}(x, h)=\left\langle z^{*}, h\right\rangle=\sum_{k=1}^{N} \lambda_{k}\left\langle z_{k}^{*}, h\right\rangle \leq \sum_{k=1}^{N} \lambda_{k} f_{i_{k}}^{\prime}(x, h) \leq \max _{i \in I_{f}(x)} f_{i}^{\prime}(x, h) .
$$

Hence (35) follows from (36) and the above inequality. The proof is complete.

The following theorem gives characterizations (by directional derivatives) of stability of local error bounds for system (30).

Theorem 13. Let $\bar{x} \in \mathbb{R}^{m}$ be such that $f(\bar{x})=0$. Then the following statements are equivalent:

i. $\inf _{\|h\|=1} f^{\prime}(\bar{x}, h) \neq 0$.

ii. There exist constants $c, \varepsilon>0$ such that if

$G \in C\left(I \times \mathbb{R}^{m}, \mathbb{R}\right), g_{i}(x):=G(i, x), g_{i}$ is convex;

$g(x):=\max _{i \in I} g_{i}(x), I_{g}(x):=\left\{i \in I: g_{i}(x)=g(x)\right\} ;$

$g(\bar{x})=0$

$I_{g}(\bar{x}) \subseteq I_{f}(\bar{x})$ whenever $\inf _{\|h\|=1} f^{\prime}(\bar{x}, h)<0$;

$I_{f}(\bar{x}) \subseteq I_{g}(\bar{x})$ whenever $\inf _{\|h\|=1} f^{\prime}(\bar{x}, h)>0 ;$

$\limsup _{x \rightarrow \bar{x}} \frac{\left|f_{i}(x)-g_{i}(x)-\left(f_{i}(\bar{x})-g_{i}(\bar{x})\right)\right|}{\|x-\bar{x}\|} \leq \varepsilon, \forall i \in I_{f}(\bar{x}) \cap I_{g}(\bar{x})$,

then one has $\tau_{\min }(G, \bar{x}) \leq c$.

iii. There exist constants $c, \varepsilon>0$ such that for all $u^{*} \in \mathbb{R}^{m}$ with $\left\|u^{*}\right\| \leq 1$, one has $\tau_{\min }(G, \bar{x}) \leq c$, where $G \in C\left(I \times \mathbb{R}^{m}, \mathbb{R}\right)$ is defined by

$G(i, x):=f_{i}(x)+\varepsilon\left\langle u^{*}, x-\bar{x}\right\rangle$ for all $(i, x) \in I \times \mathbb{R}^{m}$.

Proof. We set

$$
\beta(f, \bar{x}):=\inf _{\|h\|=1} f^{\prime}(\bar{x}, h) .
$$

$\mathbf{i} \Rightarrow$ ii. Suppose that $\beta(f, \bar{x})>0$. Then one can verify that $S_{F}=\{\bar{x}\}$ by Remark 4 . Choose any $\varepsilon \in(0, \beta(f, \bar{x}))$. Suppose that $G, g_{i}$ and $g$ satisfy all conditions said in (ii). Then for any $i \in I_{f}(\bar{x}) \subseteq I_{g}(\bar{x})$, one has

$$
g_{i}^{\prime}(\bar{x}, h) \geq f_{i}(\bar{x}, h)-\varepsilon
$$

and it follows from Proposition 12 that

$$
\begin{aligned}
\inf _{\|h\|=1} g^{\prime}(\bar{x}, h)=\inf _{\|h\|=1} \max _{i \in I_{g}(\bar{x})} g_{i}^{\prime}(\bar{x}, h) & \geq \inf _{\|h\|=1} \max _{i \in I_{f}(\bar{x})} g_{i}^{\prime}(\bar{x}, h) \\
& \geq\left(\inf _{\|h\|=1} \max _{i \in I_{f}(\bar{x})} f_{i}^{\prime}(\bar{x}, h)\right)-\varepsilon \\
& =\inf _{\|h\|=1} f^{\prime}(\bar{x}, h)-\varepsilon \\
& =\beta(f, \bar{x})-\varepsilon>0
\end{aligned}
$$


(thanks to $I_{f}(\bar{x}) \subseteq I_{g}(\bar{x})$ ). Applying Proposition 3, we derive the inequality

$$
\tau_{\min }(G, \bar{x})=\tau_{\min }(g, \bar{x}) \leq \frac{1}{\beta(f, \bar{x})-\varepsilon} .
$$

Suppose that $\beta(f, \bar{x})<0$. Choose any $\varepsilon>0$ such that $\beta(f, \bar{x})+\varepsilon<0$. Then for any $i \in I_{g}(\bar{x}) \subseteq I_{f}(\bar{x})$, one has

$$
g_{i}^{\prime}(\bar{x}, h) \leq f_{i}(\bar{x}, h)+\varepsilon
$$

and it follows from Proposition 12 that

$$
\begin{aligned}
\inf _{\|h\|=1} g^{\prime}(\bar{x}, h)=\inf _{\|h\|=1} \max _{i \in I_{g}(\bar{x})} g_{i}^{\prime}(\bar{x}, h) & \leq \inf _{\|h\|=1} \max _{i \in I_{g}(\bar{x})}\left(f_{i}^{\prime}(\bar{x}, h)+\varepsilon\right) \\
& \leq\left(\inf _{\|h\|=1} \max _{i \in I_{f}(\bar{x})} f_{i}^{\prime}(\bar{x}, h)\right)+\varepsilon \\
& =\inf _{\|h\|=1} f^{\prime}(\bar{x}, h)+\varepsilon \\
& =\beta(f, \bar{x})+\varepsilon<0
\end{aligned}
$$

(thanks to $I_{g}(\bar{x}) \subseteq I_{f}(\bar{x})$ ). Applying Proposition 3 again, we obtain the inequality

$$
\tau_{\min }(G, \bar{x})=\tau_{\min }(g, \bar{x}) \leq \frac{1}{-\beta(f, \bar{x})-\varepsilon} .
$$

ii $\Rightarrow$ iii. The implication follows immediately as $I_{f}(\bar{x})=I_{g}(\bar{x})$.

iii $\Rightarrow \mathbf{i}$. Let $u^{*} \in \mathbb{R}^{m}$ with $\left\|u^{*}\right\| \leq 1$ and $G \in C\left(I \times \mathbb{R}^{m}, \mathbb{R}\right)$ be defined as (38). Note that

$$
g_{i}(x)=G(i, x)=f_{i}(x)+\varepsilon\left\langle u^{*}, x-\bar{x}\right\rangle
$$

and thus

$$
g(x)=\max _{i \in I} g_{i}(x)=\max _{i \in I}\left(f_{i}(x)+\varepsilon\left\langle u^{*}, x-\bar{x}\right\rangle\right)=f(x)+\varepsilon\left\langle u^{*}, x-\bar{x}\right\rangle .
$$

This means that the implication follows from iii $\Rightarrow \mathrm{i}$ as in Theorem 5 . The proof is complete.

Remark 14. (a). Theorem 13, given in terms of directional derivatives, can be regarded as an equivalent version and a supplement of [42, Theorem 4] in which a subdifferential characterization of stability of local error bounds for system (30) was established. Further, in contrast with [42, Theorem 4], the stability of local error bounds for system (30) only requires that all component functions in system (30) have the same $\varepsilon$-linear perturbation.

(b). It should be observed that the condition $I_{f}(\bar{x}) \subseteq I_{g}(\bar{x})$ or $I_{g}(\bar{x}) \subseteq I_{f}(\bar{x})$ in Theorem 13 is crucial. To see this, we consider the following two examples:

ム. Let $f_{i}: \mathbb{R}^{2} \rightarrow \mathbb{R}$ be defined by $f_{i}(x):=\left|x_{i}\right|, i=1,2$ for all $x=\left(x_{1}, x_{2}\right) \in \mathbb{R}^{2}, \bar{x}=(0,0), F:=\left(f_{1}, f_{2}\right)$ and $f:=\max \left\{f_{1}, f_{2}\right\}$. Then

$$
I_{f}(\bar{x})=\{1,2\} \quad \text { and } \quad \inf _{\|h\|=1} f^{\prime}(\bar{x}, h)=\frac{\sqrt{2}}{2}>0 .
$$

However, for each $\varepsilon>0$, we define functions $g_{1, \varepsilon}$ and $g_{2, \varepsilon}$ by

$$
g_{1, \varepsilon}(x):=\left|x_{1}\right|+\varepsilon\left|x_{2}\right|, \quad g_{2, \varepsilon}(x):=\left|x_{2}\right|-\varepsilon, \text { for all } x=\left(x_{1}, x_{2}\right) \in \mathbb{R}^{2} .
$$

We set $G_{\varepsilon}:=\left(g_{1, \varepsilon}, g_{2, \varepsilon}\right)$ and $g_{\varepsilon}:=\max \left\{g_{1, \varepsilon}, g_{2, \varepsilon}\right\}$. Then one can verify that $I_{g}(\bar{x})=\{1\}$ and thus $I_{f}(\bar{x}) \not I_{g}(\bar{x})$. Note that

$$
S_{G_{\varepsilon}}=\{\bar{x}\} \quad \text { and } \operatorname{Lip}\left(f_{1}-g_{1, \varepsilon}\right) \leq \varepsilon .
$$

For any $\delta \in\left(0, \varepsilon^{-1}\right)$, we set $z_{\delta}:=(0, \delta) \in \mathbb{R}^{2}$. Then $d\left(z_{\delta}, S_{G_{\varepsilon}}\right)=\delta$ and $g_{\varepsilon}\left(z_{\delta}\right)=\varepsilon \delta$, which implies that $\tau_{\min }\left(G_{\varepsilon}, \bar{x}\right) \geq \frac{1}{\varepsilon}$. 
$\boldsymbol{\Delta} \mathbf{\Delta}$. Let $f_{1}, f_{2}: \mathbb{R}^{2} \rightarrow \mathbb{R}$ be defined by $f_{1}(x):=x_{1}$ and $f_{1}(x):=-x_{1}+\left|x_{2}\right|-1$ for all $x=\left(x_{1}, x_{2}\right) \in \mathbb{R}^{2}$, $\bar{x}=(0,0), F:=\left(f_{1}, f_{2}\right)$ and $f:=\max \left\{f_{1}, f_{2}\right\}$. Then

$$
I_{f}(\bar{x})=\{1\} \quad \text { and } \quad \inf _{\|h\|=1} f^{\prime}(\bar{x}, h)=\inf _{\|h\|=1} f_{1}^{\prime}(\bar{x}, h)=-1<0 .
$$

However, for each $\varepsilon>0$, we define functions $g_{1, \varepsilon}$ and $g_{2, \varepsilon}$ as

$$
g_{1, \varepsilon}(x):=x_{1}+\varepsilon\left|x_{2}\right|, g_{2, \varepsilon}(x):=-x_{1}+\varepsilon\left|x_{2}\right|, \text { for all } x=\left(x_{1}, x_{2}\right) \in \mathbb{R}^{2} .
$$

We set $G_{\varepsilon}:=\left(g_{1, \varepsilon}, g_{2, \varepsilon}\right)$ and $g_{\varepsilon}:=\max \left\{g_{1, \varepsilon}, g_{2, \varepsilon}\right\}$. Then one can verify that $I_{g}(\bar{x})=\{1,2\}$ and thus $I_{g}(\bar{x}) \nsubseteq I_{f}(\bar{x})$. Note that

$$
S_{G_{\varepsilon}}=\{\bar{x}\} \quad \text { and } \operatorname{Lip}\left(f_{1}-g_{1, \varepsilon}\right) \leq \varepsilon .
$$

For any $\delta \in\left(0, \varepsilon^{-1}\right)$, set $z_{\delta}:=(0, \delta) \in \mathbb{R}^{2}$. Then $d\left(z_{\delta}, S_{G_{\varepsilon}}\right)=\delta$ and $g_{\varepsilon}\left(z_{\delta}\right)=\varepsilon \delta$. This means that $\tau_{\min }\left(G_{\varepsilon}, \bar{x}\right) \geq$ $\varepsilon^{-1}$.

We now turn our attention to the stability of global error bounds for semi-infinite constraint system (30) and mainly give equivalent criterion for such stability. Based on Theorem 10, the following theorem establishes equivalent conditions for the stability of global error bounds for the system (30).

- Theorem 15. The following statements are equivalent:

i. There exists $\tau \in(0,+\infty)$ such that

$\inf \left\{\left|\inf _{\|h\|=1} f^{\prime}(\bar{x}, h)\right|: \bar{x} \in \operatorname{bdry}\left(S_{f}\right)\right\}>\tau$,

and (QC) as in Theorem 10 is satisfied.

ii. There exist constants $c, \varepsilon>0$ such that if

$G, g_{i}(x), g(x)$ and $I_{g}(x)$ as said in Theorem 13.ii;

$\left\{z \in \operatorname{bdry}\left(S_{f}\right): f_{i}(z)=g_{i}(z)\right.$ for all $\left.i \in I\right\} \neq \emptyset$;

$\sup _{i \in I} \operatorname{Lip}\left(f_{i}-g_{i}\right)<\varepsilon$

$I_{g}(x) \subseteq I_{f}(x)$ whenever $\inf _{\|h\|=1} f^{\prime}(x, h)<0$;

$I_{f}(x) \subseteq I_{g}(x)$ whenever $\inf _{\|h\|=1} f^{\prime}(x, h)>0$,

then one has $\tau_{\min }(G) \leq c$.

iii. There exist constants $c, \varepsilon>0$ such that for all $\bar{x} \in \operatorname{bdry}\left(S_{f}\right)$ and $u^{*} \in \mathbb{R}^{m}$ with $\left\|u^{*}\right\| \leq 1$, one has $\tau_{\min }(G) \leq c$, where $G \in C\left(I \times \mathbb{R}^{m}, \mathbb{R}\right)$ is defined by

$G(i, x):=f_{i}(x)+\varepsilon\left\langle u^{*}, x-\bar{x}\right\rangle$ for all $(i, x) \in I \times \mathbb{R}^{m}$.

Proof. $\mathbf{i} \Rightarrow$ ii. Thanks to Remark 4 and the proof of Proposition 13, we only need to consider the case $\inf _{\|h\|=1} f^{\prime}(\bar{x}, h) \leq 0$ for all $\bar{x} \in \operatorname{bdry}\left(S_{f}\right)$. By virtue of the claim given in the proof of Theorem 10, there exists $\varepsilon_{0}>0$ such that for all $x_{0} \in \operatorname{bdry}\left(S_{f}\right)$, one has

$$
\inf \left\{\left|\inf _{\|h\|=1} f^{\prime}(x, h)\right|: x \in \mathbb{R}^{m}, f(x) \geq-\varepsilon_{0}\left\|x-x_{0}\right\|\right\} \geq \tau .
$$

Take any $\varepsilon>0$ such that $\varepsilon<\min \left\{\varepsilon_{0}, \tau\right\}$. Suppose that $G, g_{i}$ and $g$ satisfy all conditions said in ii. Let $x \in \mathbb{R}^{m}$ be such that $g(x)>0$. We claim that

$$
\inf _{\|h\|=1} f^{\prime}(x, h) \leq-\tau .
$$

Granting this, one has

$$
\begin{aligned}
\inf _{\|h\|=1} g^{\prime}(x, h)=\inf _{\|h\|=1}\left(\max _{i \in I_{g}(x)} g_{i}^{\prime}(x, h)\right) & \leq \inf _{\|h\|=1}\left(\max _{i \in I_{g}(x)} f_{i}^{\prime}(x, h)+\varepsilon\right) \\
& \leq \inf _{\|h\|=1}\left(\max _{i \in I_{f}(x)} f_{i}^{\prime}(x, h)\right)+\varepsilon \\
& =\inf _{\|h\|=1} f^{\prime}(x, h)+\varepsilon \\
& \leq-(\tau-\varepsilon)
\end{aligned}
$$


(thanks to $\sup _{i \in I} \operatorname{Lip}\left(f_{i}-g_{i}\right)<\varepsilon$ and $I_{g}(x) \subseteq I_{f}(x)$ ). Applying Lemma 1 and Theorem 2, we derive the inequality $\tau_{\min }(G) \leq \frac{1}{\tau-\varepsilon}$.

It remains to prove relation (42). For the case that $f(x)>0$, similar to the proof of (19), one can verify that (42) holds. Thus we only need to consider the case that $f(x) \leq 0$.

From the second condition in (ii), there is $z_{0} \in \operatorname{bdry}\left(S_{f}\right)$ such that $f_{i}\left(z_{0}\right)=g_{i}\left(z_{0}\right)$ for all $i \in I$. Then for any $i \in I_{g}(x) \subseteq I_{f}(x)$, one has

$$
f_{i}(x) \geq g_{i}(x)-\left(f_{i}\left(z_{0}\right)-g_{i}\left(z_{0}\right)\right)-\varepsilon\left\|x-z_{0}\right\|=g(x)-\varepsilon\left\|x-z_{0}\right\|>-\varepsilon\left\|x-z_{0}\right\|
$$

and thus $f(x)>-\varepsilon_{0}\left\|x-z_{0}\right\|$. This and (41) imply that (42) holds.

ii $\Rightarrow$ iii. The implication follows immediately since $I_{f}(x)=I_{g}(x)$ for all $x \in \mathbb{R}^{m}$.

iii $\Rightarrow \mathbf{i}$. Let $\bar{x} \in \operatorname{bdry}\left(S_{f}\right), u^{*} \in \mathbb{R}^{m}$ with $\left\|u^{*}\right\| \leq 1$ and $G \in C\left(I \times \mathbb{R}^{m}, \mathbb{R}\right)$ be defined as (40). Note that

$$
g_{i}(x)=G(i, x)=f_{i}(x)+\varepsilon\left\langle u^{*}, x-\bar{x}\right\rangle
$$

and consequently

$$
g(x)=\max _{i \in I} g_{i}(x)=\max _{i \in I}\left(f_{i}(x)+\varepsilon\left\langle u^{*}, x-\bar{x}\right\rangle\right)=f(x)+\varepsilon\left\langle u^{*}, x-\bar{x}\right\rangle .
$$

Thus, the implication follows from iii $\Rightarrow \mathrm{i}$ as in Theorem 10 . The proof is complete

\section{Conclusions}

This paper is devoted to the study of stability of local and global error bounds for convex inequality constraint systems including a single convex inequality and semi-infinite convex constraint systems. The main results provide characterizations (in terms of directional derivatives) of stability of local and global error bounds for a single convex inequality (see Theorem 5 and Theorem 10). When these results are applied to error bounds for semi-infinite convex constraint systems, characterizations of the stability of local and global error bounds are also established in terms of directional derivatives (see Theorem 13 and Theorem 15). These results show that the stability of error bounds for convex inequality constraint systems can be equivalent to solving the convex optimization/minimization problems defined by directional derivatives over the unit sphere.

\section{References}

1 Malek Abbasi and Michel Théra. Strongly regular points of mappings. Fixed Point Theory Algorithms Sci. Eng., 2021, 2021.

2 A. A. Auslender and Jean-Pierre Crouzeix. Global regularity theorems. Math. Oper. Res., 13(2):243-253, 1988.

3 Dominique Azé. A survey on error bounds for lower semicontinuous functions. ESAIM, Proc., 13:1-17, 2003. Proceedings of 2003 MODE-SMAI Conference.

4 Dominique Azé. A unified theory for metric regularity of multifunctions. J. Convex Anal., 13(2):225-252, 2006.

5 Dominique Azé and Jean-Noël Corvellec. On the sensitivity analysis of Hoffman constants for systems of linear inequalities. SIAM J. Optim., 12(4):913-927, 2002.

6 Dominique Azé and Jean-Noël Corvellec. Characterizations of error bounds for lower semicontinuous functions on metric spaces. ESAIM, Control Optim. Calc. Var., 10:409-425, 2004.

7 Heinz H. Bauschke and Jonathan M. Borwein. On projection algorithms for solving convex feasibility problems. SIAM Rev., 38(3):367-426, 1996.

8 Amir Beck and Marc Teboulle. Convergence rate analysis and error bounds for projection algorithms in convex feasibility problems. Optim. Methods Softw., 18(4):377-394, 2003.

9 Ewa M. Bednarczuk and Alexander Y. Kruger. Error bounds for vector-valued functions: necessary and sufficient conditions. Nonlinear Anal., Theory Methods Appl., 75(3):1124-1140, 2012.

10 James V. Burke and Sien Deng. Weak sharp minima revisited. I. Basic theory. Control Cybern., 31(3):439-469, 2002.

11 James V. Burke and Sien Deng. Weak sharp minima revisited. II. Application to linear regularity and error bounds. Math. Program., 104(2-3):235-261, 2005.

12 María J. Cánovas, Alexander Y. Kruger, Marco A. López, Juan Parra, and Michel Théra. Calmness modulus of linear semi-infinite programs. SIAM J. Optim., 24(1):29-48, 2014.

13 Patrick L. Combettes. Hilbertian convex feasibility problem: convergence of projection methods. Appl. Math. Optim., 35(3):311-330, 1997. 
14 Jean-Noël Corvellec and Viorica V. Motreanu. Nonlinear error bounds for lower semicontinuous functions on metric spaces. Math. Program., 114(2):291-319, 2008.

15 Nguyen Duy Cuong and Alexander Y. Kruger. Error bounds revisited. https://arxiv.org/abs/2012.03941v1, 2020.

16 Sien Deng. Perturbation analysis of a condition number for convex inequality systems and global error bounds for analytic systems. Math. Program., 83(2):263-276, 1998.

17 Asen L. Dontchev, Adrian S. Lewis, and Ralph T. Rockafellar. The radius of metric regularity. Trans. Am. Math. Soc., 355(2):493-517, 2003.

18 Marian J. Fabian, René Henrion, Alexander Y. Kruger, and Jiří V. Outrata. Error bounds: necessary and sufficient conditions. Set-Valued Var. Anal., 18(2):121-149, 2010.

19 Helmut Gfrerer. First order and second order characterizations of metric subregularity and calmness of constraint set mappings. SIAM J. Optim., 21(4):1439-1474, 2011.

20 Osman Güler. Augmented Lagrangian algorithms for linear programming. J. Optim. Theory Appl., 75(3):445-478, 1992.

21 Robert Hesse and D. Russell Luke. Nonconvex notions of regularity and convergence of fundamental algorithms for feasibility problems. SIAM J. Optim., 23(4):2397-2419, 2013.

22 A. J. Hoffman. On approximate solutions of systems of linear inequalities. J. Res. Nat. Bur. Standards, 49:263-265, 1952 .

23 L. R. Huang and Kung Fu Ng. On first- and second-order conditions for error bounds. SIAM J. Optim., 14(4):10571073, 2004.

24 Alexander D. Ioffe. Theory of Extremal Problems, volume 6 of Studies in Mathematics and its Applications. North-Holland, 1979.

25 Alexander D. Ioffe. Metric regularity - a survey. I: Theory. J. Aust. Math. Soc., 101(2):188-243, 2016.

26 Alexander D. Ioffe. Metric regularity - a survey. II: Applications. J. Aust. Math. Soc., 101(3):376-417, 2016.

27 Alexander D. Ioffe. Variational analysis of regular mappings. Theory and applications. Springer Monographs in Mathematics. Springer, 2017.

28 Alfredo N. Iusem and Alvaro R. De Pierro. On the convergence properties of Hildreth's quadratic programming algorithm. Math. Program., 47(1):37-51, 1990.

29 Abderrahim Jourani. Hoffman's error bound, local controllability, and sensitivity analysis. SIAM J. Control Optimization, 38(3):947-970, 2000.

30 Diethard Klatte and Wu Li. Asymptotic constraint qualifications and global error bounds for convex inequalities. Math. Program., 84(1):137-160, 1999.

31 Alexander Y. Kruger. Error bounds and Hölder metric subregularity. Set-Valued Var. Anal., 23(4):705-736, 2015.

32 Alexander Y. Kruger. Error bounds and metric subregularity. Optimization, 64(1):49-79, 2015.

33 Alexander Y. Kruger, Marco A. López, and Michel Théra. Perturbation of error bounds. Math. Program., 168(12):533-554, 2018 .

34 Alexander Y. Kruger, Marco A. López, Xiaoqi Yang, and Jiangxing Zhu. Hölder error bounds and Hölder calmness with applications to convex semi-infinite optimization. Set-Valued Var. Anal., 27(4):995-1023, 2019.

35 Alexander Y. Kruger, Huynh Van Ngai, and Michel Théra. Stability of error bounds for convex constraint systems in Banach spaces. SIAM J. Optim., 20(6):3280-3296, 2010.

36 Adrian S. Lewis and Jong-Shi Pang. Error bounds for convex inequality systems. In Generalized Convexity, Generalized Monotonicity: Recent Results (Luming, 1996), volume 27 of Nonconvex Optimization and Its Applications, pages 75-100. Kluwer Academic Publishers, 1996.

37 D. Russell Luke, H. Thao Nguyen, and Matthew K. Tam. Implicit error bounds for Picard iterations on Hilbert spaces. Vietnam J. Math., 46(2):243-258, 2018.

38 Zhi-Quan Luo and Paul Tseng. On a global error bound for a class of monotone affine variational inequality problems. Oper. Res. Lett., 11(3):159-165, 1992.

39 Zhi-Quan Luo and Paul Tseng. Perturbation analysis of a condition number for linear systems. SIAM J. Matrix Anal. Appl., 15(2):636-660, 1994.

40 Olvi L. Mangasarian. A condition number for differentiable convex inequalities. Math. Oper. Res., 10:175-179, 1985.

41 Kung Fu Ng and Xi Yin Zheng. Error bounds for lower semicontinuous functions in normed spaces. SIAM J. Optim., 12(1):1-17, 2001.

42 Huynh Van Ngai, Alexander Y. Kruger, and Michel Théra. Stability of error bounds for semi-infinite convex constraint systems. SIAM J. Optim., 20(4):2080-2096, 2080.

43 Huynh Van Ngai and Michel Théra. Error bounds for systems of lower semicontinuous functions in Asplund spaces. Math. Program., 116(1-2):397-427, 2009.

44 Jong-Shi Pang. Error bounds in mathematical programming. Math. Program., 79(1-3):299-332, 1997.

45 Jean-Paul Penot. Calculus without derivatives, volume 266 of Graduate Texts in Mathematics. Springer, 2013.

46 Robert R. Phelps. Convex functions, Monotone Operators and Differentiability, volume 1364 of Lecture Notes in 
Mathematics. Springer, 2nd edition, 1993.

47 Stephen M. Robinson. Bounds for error in the solution set of a perturbed linear program. Linear Algebra Appl., 6:69-81, 1973.

48 Stephen M. Robinson. An application of error bounds for convex programming in a linear space. SIAM J. Control, 13:271-273, 1975.

49 Stephen M. Robinson. A characterization of stability in linear programming. Oper. Res., 25:435-447, 1977.

50 Ralph T. Rockafellar. Convex Analysis, volume 28 of Princeton Mathematical Series. Princeton University Press, 1970.

51 Paul Tseng and Dimitri P. Bertsekas. On the convergence of the exponential multiplier method for convex programming. Math. Program., 60(1):1-19, 1993.

52 Zili Wu and Jane J. Ye. On error bounds for lower semicontinuous functions. Math. Program., 92(2):301-314, 2002.

$53 \mathrm{Xi}$ Yin Zheng and Kung Fu Ng. Perturbation analysis of error bounds for systems of conic linear inequalities in Banach spaces. SIAM J. Optim., 15(4):1026-1041, 2005.

54 Xi Yin Zheng and Kung Fu Ng. Metric subregularity and calmness for nonconvex generalized equations in Banach spaces. SIAM J. Optim., 20(5):2119-2136, 2010.

$55 \mathrm{Xi}$ Yin Zheng and Kung Fu Ng. Metric subregularity for proximal generalized equations in Hilbert spaces. Nonlinear Anal., Theory Methods Appl., 75(3):1686-1699, 2012.

$56 \mathrm{Xi}$ Yin Zheng and Zhou Wei. Perturbation analysis of error bounds for quasi-subsmooth inequalities and semi-infinite constraint systems. SIAM J. Optim., 22(1):41-65, 2012. 